\title{
Magnon Condensation and Spin Superfluidity
}

\author{
Yury M. Bunkov ${ }^{a}$ and Vladimir L. Safonov ${ }^{b, c}$ \\ (a) Kazan Federal University, Kremlevskaya 18, 420008 Kazan, Russia \\ (b) Mag and Bio Dynamics, Inc., Granbury, TX 76049, USA \\ (c) Physical Science Department, Tarrant County \\ College - South Campus, Fort Worth, TX 76119, USA
}

\begin{abstract}
We consider the Bose-Einstein condensation (BEC) of quasi-equilibrium magnons which leads to spin superfluidity, the coherent quantum transfer of magnetization in magnetic material. The critical conditions for excited magnon density in ferro- and antiferromagnets, bulk and thin films, are estimated and discussed. It was demonstrated that only the highly populated region of the spectrum is responsible for the emergence of any BEC. This finding substantially simplifies the BEC theoretical analysis and is surely to be used for simulations. It is shown that the conditions of magnon BEC in the perpendicular magnetized YIG thin film is fulfillied at small angle, when signals are treated as excited spin waves. We also predict that the magnon BEC should occur in the antiferromagnetic hematite at room temperature at much lower excited magnon density compared to that of ferromagnetic YIG. Bogoliubov's theory of Bose-Einstein condensate is generalized to the case of multi-particle interactions. The six-magnon repulsive interaction may be responsible for the BEC stability in ferro- and antiferromagnets where the four-magnon interaction is attractive.
\end{abstract}

PACS numbers: $75.45 .+\mathrm{j}$

Keywords: Bose-Einstein condensation, magnons, YIG, hematite 


\section{INTRODUCTION}

Spin deviations from the magnetic order in a magnetic material (ferromagnet, antiferromagnet or ferrites) are manifested by spin waves and their quanta, magnons. Magnons are quasiparticles which represent a very useful quantum theoretical tool to describe various dynamic and thermodynamic processes in magnets in terms of magnon gas. Since magnons have magnetic moments, the external alternating magnetic field can excite extra magnons and increase the disorder in the magnetic system. However, in certain conditions, the increase of magnon density leads to a new state, so-called, magnon condensate, in which a macroscopic number of magnons forms a coherent quantum state (see, e.g., [1], [2]). This macroscopic state can significantly change the properties of magnon gas, its dynamics and transport. An example is the phenomenon of quasi-equilibrium Bose-Einstein condensation (BEC) of excited magnons on the bottom of their spectrum as a single-particle long-range coherent state of quantum liquid. This state generate an uniform long-lived precession of spins formed by quantum specificity of the magnon gas when the magnon density exceeds certain critical value. The spatial gradients of this state exhibit a spin superfluidity, the non-potential transport of deflected magnetization. The spin superfluidity is an extremely interesting phenomenon for both fundamental and applied studies. It should be emphasized that the main paradigm of magnetic dynamics, the Landau-Lifshitz-Gilbert equation, does not contain complete information about the Bose-Einstein condensate of magnons. BEC is the principal result of quantum statistics and for magnons it can exist at room and even higher temperatures.

For the first time the existence of quasi-equilibrium Bose condensate was demonstrated in the experiment with nuclear magnons in the superfluid antiferromagnetic liquid crystal ${ }^{3} \mathrm{He}-\mathrm{B}$ in 1984 [3]. The theoretical explanation of this phenomenon [4] was developed on the basis of global Ginzburg-Landau energy potential. A similar approach was later developed to explain the atomic BEC [5]. In the experiments with an antiferromagnetic ${ }^{3} \mathrm{He}-\mathrm{B}$, the following phenomena were observed: a) transport of magnetization by spin supercurrent between two cells with magnon BEC; b) phase-slip processes at the critical current; c) spin current Josephson effect; d) spin current vortex formation; d) Goldstone modes of magnon BEC oscillations. Comprehensive reviews of these studies can be found in Refs. 6 8]. Currently magnon BEC found in different magnetic systems: i) in antiferromagnetic 
superfluid ${ }^{3} \mathrm{He}-\mathrm{A}$ [9, 10]; ii) in in-plane magnetized yttrium iron garnet $\mathrm{Y}_{3} \mathrm{Fe}_{5} \mathrm{O}_{12}$ (YIG) film (with two minima in the magnon spectrum) [11, 12] and in normally magnetized YIG film [13]; iii) in antiferromagnets $\mathrm{MnCO}_{3}$ and $\mathrm{CsMnF}_{3}$ with Suhl-Nakamura indirect nuclear spin-spin interaction [14-16]. An explanation of analogy between the atomic and magnon BEC is given in Ref. [17].

A microscopic theory of quasi-equilibrium magnon BEC was developed in Refs.[18]-[21] ("KS theory"). It was predicted that the external strong pumping of magnons leads to a rapid growth of magnon density and saturation. This state can be considered in terms of weakly non-ideal gas of "dressed" magnons in a thermodynamic quasi-equilibrium with an effective chemical potential $\mu$ and effective temperature $T$. The dressed magnon energy is defined by $\varepsilon_{\mathbf{k}}=\varepsilon_{\mathbf{k}}^{(0)}+\delta \varepsilon_{\mathbf{k}}$, where $\varepsilon_{\mathbf{k}}^{(0)}=\hbar \omega_{\mathbf{k}}$ is the energy spectrum of bare magnons and $\delta \varepsilon_{\mathbf{k}}$ is the energy shift due to magnon gas nonlinearities. Magnon-magnon scattering processes retain the total number of dressed magnons in the system and hold their distribution function of the form

$$
n_{\mathbf{k}}=\left(\exp \frac{\varepsilon_{\mathbf{k}}-\mu}{k_{B} T}-1\right)^{-1}
$$

The instability at $\mu=\min \varepsilon_{\mathbf{k}}$ in the quasi-equilibrium magnetic system is an analog of BEC phenomenon for the bottom dressed magnons. The distribution (1) seems to underlie the phenomenon of spin superfluidity, since it nullifies the integral of four-magnon collisions

$$
\begin{aligned}
I^{(4)}\left\{n_{\mathbf{k}}\right\} \propto & \int d^{3} k_{1} d^{3} k_{2} d^{3} k_{3}\left|\Phi_{4}\left(\mathbf{k}, \mathbf{k}_{1} ; \mathbf{k}_{2}, \mathbf{k}_{3}\right)\right|^{2} \\
& \times\left[\left(n_{\mathbf{k}}+1\right)\left(n_{\mathbf{k}_{1}}+1\right) n_{\mathbf{k}_{3}} n_{\mathbf{k}_{4}}-n_{\mathbf{k}} n_{\mathbf{k}_{1}}\left(n_{\mathbf{k}_{2}}+1\right)\left(n_{\mathbf{k}_{3}}+1\right)\right] \\
& \times \delta\left(\varepsilon_{\mathbf{k}}+\varepsilon_{\mathbf{k}_{1}}-\varepsilon_{\mathbf{k}_{2}}-\varepsilon_{\mathbf{k}_{3}}\right) \Delta\left(\mathbf{k}+\mathbf{k}_{1}-\mathbf{k}_{2}-\mathbf{k}_{3}\right)
\end{aligned}
$$

and thus this energy loss channel vanishes.

KS theory qualitatively explained the parallel pumping experiments ([22],[23] (YIG at room temperature) and [24] (nuclear magnons in $\mathrm{CsMnF}_{3}$ ), where the accumulation of magnons at the bottom of the spin wave spectrum was observed. One and a half decade later, purposeful experiment [11] directly demonstrated BEC of quasi equilibrium magnons in the thin film of YIG. Subsequent experimental studies have shown qualitative correspondence with the predicted distribution of excited magnons [25] and agreement with the BEC under noisy pumping [26]. 
In this paper we analyze critical conditions of quasi-equilibrium magnon BEC in ferroand antiferromagnets, bulk and thin films, and evaluate the possibilities of their experimental achievements.

\section{BEC OF BOSE PARTICLES}

Let us first briefly discuss BEC of real bose particles. Their distribution is defined by Eq.(11), where $\varepsilon_{k}=(\hbar k)^{2} / 2 m$ is the kinetic energy of particle with the wave vector $k$ and mass $m$. The total number of bosons in the system is

$$
N(\mu, T)=N=V_{s} \int n_{\mathbf{k}} \frac{d^{3} k}{(2 \pi)^{3}},
$$

where $V_{s}$ is the volume of the system. For the critical condition $\mu=\min \varepsilon_{k}$, from (3) follows well-known formula for the BEC critical temperature versus the density of bosons:

$$
T_{B E C}=\kappa_{0} \frac{\hbar^{2}}{k_{B} m}\left(\frac{N}{V_{s}}\right)^{2 / 3}, \quad \kappa_{0}=\frac{2 \pi}{\left[\zeta\left(\frac{3}{2}\right)\right]^{2 / 3}} \simeq 3.31
$$

It is interesting to note that the BEC is formed mainly by bosons with high populations when Eq.(1) can be written as

$$
n_{k} \simeq \frac{k_{B} T}{\varepsilon_{k}-\mu}
$$

Let us prove it by direct calculation. Substituting the high temperature population (5) into Eq.(3) and cutting the upper integral limit by the thermal energy $\varepsilon_{T} \simeq k_{B} T$, one obtains:

$$
T_{B E C} \simeq \tilde{\kappa}_{0} \frac{\hbar^{2}}{k_{B} m}\left(\frac{N}{V_{s}}\right)^{2 / 3}, \quad \tilde{\kappa}_{0}=\frac{\pi^{4 / 3}}{2^{1 / 3}} \simeq 3.65 .
$$

We see that the only difference between Eqs.(44) and (6) is a slightly different $(\sim 10 \%)$ numerical factor.

The fact that the high population Eq.(5) is dominant does not mean that the BEC phenomenon is a classical one. The criterion of classical Maxwell-Boltzmann statistics $\exp \left(\mu / k_{B} T\right) \ll 1$ in this case can be written as (see, e.g., [27]):

$$
\exp \left(\mu / k_{B} T\right)=\left[\frac{V_{s}}{N} \int \exp \left(-\frac{\varepsilon_{\mathbf{k}}}{k_{B} T}\right) \frac{d^{3} k}{(2 \pi)^{3}}\right]^{-1} \ll 1
$$

or 


$$
\frac{N}{V_{s}} \lambda_{T}^{3}=\frac{N}{V_{s}}\left(\frac{2 \pi \hbar^{2}}{m k_{B} T}\right)^{3 / 2} \ll 1,
$$

where $\lambda_{T}$ is the thermal de Broglie wavelength. Substituting BEC temperature Eq.(6) into (8), we obtain the opposite relation: $2.26>1$, which obviously corresponds to a degenerate bose gas.

\section{BEC OF MAGNONS}

Now let us consider a Bose-Einstein condensation of so-called, "dressed" magnons as an instability in the externally pumped quasi-equilibrium magnon gas. The total number of magnons $N(\mu, T)$ is equal to the number of thermal magnons $N(0, T)$ at a given temperature $T$ and the number of magnons $N_{p}$ created by external pumping. So far as the energy shift

of dressed magnons is usually much less than the energy of bare magnons $\delta \varepsilon_{k} \ll \min \varepsilon_{k}^{(0)}$, for simplicity we can approximate $\varepsilon_{k} \simeq \varepsilon_{k}^{(0)}$.

\section{$\mathrm{BEC}$ in a ferromagnet}

Consider a ferromagnet with the quadratic spectrum (we neglect details of the dipoledipole interactions):

$$
\varepsilon_{k}=\varepsilon_{0}+\varepsilon_{e x}(a k)^{2}
$$

Here $\varepsilon_{e x}$ is the exchange interaction constant and $a$ is the elementary cell linear size. The quasi-equilibrium BEC will be mainly determined by pumping if the number of pumped magnons is much greater than the thermal magnon number $N_{p} \gg N(0, T)$. In this case we obtain an analog of Eq.(44):

$$
T_{B E C}=\kappa_{0} \frac{2 \varepsilon_{e x}}{k_{B}}\left(a^{3} \frac{N_{p}}{V_{s}}\right)^{2 / 3}
$$

or,

$$
T_{B E C} \simeq \tilde{\kappa}_{0} \frac{2 \varepsilon_{e x}}{k_{B}}\left(a^{3} \frac{N_{p}}{V_{s}}\right)^{2 / 3}
$$

in the high-population approximation. 
The above formula, however, does not work for a BEC estimate if $N_{p} \lesssim N(0, T)$. Using a high-population approximation, we write

$$
\begin{aligned}
N_{p} & =N(\mu, T)-N(0, T) \\
& \simeq V_{s} \int_{\varepsilon_{0}}^{\varepsilon_{T}}\left(\frac{k_{B} T}{\varepsilon_{k}-\mu}-\frac{k_{B} T}{\varepsilon_{k}}\right) \frac{k^{2} d k}{2 \pi^{2}},
\end{aligned}
$$

and obtain at $\mu=\varepsilon_{0}$

$$
\begin{aligned}
\frac{N_{p}}{V_{s}} & \simeq \frac{k_{B} T_{B E C}}{4 \pi a^{3}} \frac{\varepsilon_{0}^{1 / 2}}{\varepsilon_{e x}^{3 / 2}} \\
T_{B E C} & \simeq 4 \pi \frac{\varepsilon_{e x}}{k_{B}}\left(\frac{\varepsilon_{e x}}{\varepsilon_{0}}\right)^{1 / 2}\left(a^{3} \frac{N_{p}}{V_{s}}\right) .
\end{aligned}
$$

These formulas coincide with the accuracy of notations with the exact calculation given in Ref.[2]. This is one more direct proof that BEC is formed by the high-populated part of spectrum.

An estimate for YIG, where $\varepsilon_{e x} a^{2} / \hbar=0.092 \mathrm{~cm}^{2} \mathrm{~s}^{-1}$ for $\varepsilon_{0} / \hbar=2 \pi \times 2.5 \mathrm{GHz}$ gives $T_{B E C} \simeq 2.14 \times 10^{-17}\left(N_{p} / V_{s}\right) \mathrm{cm}^{3} \mathrm{~K}$. Thus, we obtain a room-temperature BEC $T_{B E C} \simeq 300$ $\mathrm{K}$ at the pumped magnon density $N_{p} / V_{s}=1.41 \times 10^{19} \mathrm{~cm}^{-3}$ that in order of magnitude corresponds to the experiment [11].

As in the above case of particles, the opposition of high density of magnons to their high population makes the classical criterion $\exp \left[\left(\mu-\varepsilon_{0}\right) / k_{B} T\right] \ll 1$ inapplicable to the case of condensation when $\mu=\varepsilon_{0}$. As in the previous section, we can write the criterion for Maxwell-Boltzmann statistics

$$
\exp \left[\left(\mu-\varepsilon_{0}\right) / k_{B} T\right]=\left[\frac{V_{s}}{N(\mu, T)} \int \exp \left(-\frac{\varepsilon_{e x}(a k)^{2}}{k_{B} T}\right) \frac{d^{3} k}{(2 \pi)^{3}}\right]^{-1} \ll 1,
$$

or

$$
\frac{N(\mu, T)}{V_{s}} \lambda_{T}^{3}=\frac{N(\mu, T)}{V_{s}}\left(\frac{4 \pi \varepsilon_{e x} a^{2}}{k_{B} T}\right)^{3 / 2} \ll 1 .
$$

In the case of high temperatures we have $N(\mu, T) \simeq N(0, T)$. Substituting the thermal density

$$
\frac{N(0, T)}{V_{s}}=\left(\frac{k_{B} T}{2 \kappa_{0} \varepsilon_{e x} a^{2}}\right)^{3 / 2}
$$


into (15), we obtain the opposite relation: $2.61>1$. In other words, magnon gas which undergoes Bose-Einstein condensation is always a degenerate bose gas. Thus the assertion of recent publication [28] "that the experimentally observed condensation of magnons in yttrium-iron garnet at room temperature is a purely classical phenomenon" is untenable.

\section{BEC in an antiferromagnet}

Consider now the magnon energy of the form

$$
\varepsilon_{k}=\sqrt{\varepsilon_{0}^{2}+\varepsilon_{e x}^{2}(a k)^{2}} .
$$

This is typical for magnons in the "easy-plane" (or, canted) antiferromagnets. Taking into account that

$$
k=\frac{\sqrt{\varepsilon_{k}^{2}-\varepsilon_{0}^{2}}}{a \varepsilon_{e x}} \quad \text { and } \quad k d k=\frac{\varepsilon_{k} d \varepsilon_{k}}{\varepsilon_{e x}^{2} a^{2}},
$$

in the high-population approximation, one can write

$$
\frac{N\left(\mu=\varepsilon_{0}, T\right)}{V_{s}} \simeq \frac{k_{B} T}{2 \pi^{2}} \frac{1}{a^{3} \varepsilon_{e x}^{3}} \int_{\varepsilon_{0}}^{\varepsilon_{T}} \sqrt{\frac{\varepsilon+\varepsilon_{0}}{\varepsilon-\varepsilon_{0}}} \varepsilon d \varepsilon .
$$

If $N_{p} \gg N(0, T)$, for $k_{B} T \gg \varepsilon_{0}$ we obtain

$$
T_{B E C} \simeq(2 \pi)^{2 / 3} \frac{\varepsilon_{e x}}{k_{B}}\left(a^{3} \frac{N_{p}}{V_{s}}\right)^{1 / 3} .
$$

If $N_{p} \lesssim N(0, T)$, one can rewrite Eq.(12) in the form:

$$
\frac{N_{p}}{V_{s}} \simeq \frac{k_{B} T}{2 \pi^{2}} \frac{\varepsilon_{0}}{a^{3} \varepsilon_{e x}^{3}} \int_{\varepsilon_{0}}^{\varepsilon_{T}} \sqrt{\frac{\varepsilon+\varepsilon_{0}}{\varepsilon-\varepsilon_{0}}} d \varepsilon
$$

After integration, at $k_{B} T \gg \varepsilon_{0}$ we obtain

$$
\frac{N_{p}}{V_{s}} \simeq \frac{\left(k_{B} T_{B E C}\right)^{2}}{2 \pi^{2}} \frac{\varepsilon_{0}}{a^{3} \varepsilon_{e x}^{3}},
$$

or,

$$
T_{B E C} \simeq \sqrt{2} \pi \frac{\varepsilon_{e x}}{k_{B}}\left(\frac{\varepsilon_{e x}}{\varepsilon_{o}}\right)^{1 / 2}\left(a^{3} \frac{N_{p}}{V_{s}}\right)^{1 / 2} .
$$

Note that the BEC temperature for antiferromagnet has lower power dependence on small parameter $a^{3} N_{p} / V_{s} \ll 1$ and therefore one can expect much lower densities of pumped magnons to achieve condensation. An estimate for $\alpha-\mathrm{Fe}_{2} \mathrm{O}_{3}$ (hematite), where $\varepsilon_{e x} a / \hbar \approx$ $24 \times 10^{5} \mathrm{~cm} / \mathrm{s}$ for $\varepsilon_{0} / \hbar=2 \pi \times 2.5 \mathrm{GHz}$ gives $T_{B E C} \approx 10^{-6}\left(N_{p} / V_{s}\right)^{1 / 2} \mathrm{~cm}^{3 / 2} \mathrm{~K}$. Thus we obtain a room-temperature BEC, $T=300 \mathrm{~K}$ at $N_{p} / V_{s}=0.89 \times 10^{17} \mathrm{~cm}^{-3}$. This estimate is more lower than corresponding estimate for a ferromagnetic YIG. This means that hematite is a very attractive object to observe $\mathrm{BEC}$ of magnons experimentally. 


\section{BEC IN A FERROMAGNETIC FILM}

Let us now consider an ultra-thin ferromagnetic film. There are two principal cases: 1) external magnetic field $\mathbf{H}$ is parallel to the the film surface and 2) $\mathbf{H}$ is perpendicular to this surface. In the first case, the BEC condition $\mu=\min \hbar \omega_{\mathbf{k}}$ gives us two minima at $\pm \mathbf{k}_{\min } \neq 0$. This case was demonstrated experimentally for the YIG film in Ref.[11], where the critical density of pumped magnons was estimated numerically. Later, in Ref.[1] it was considered analytically. Here we focus on the second case with just one energy minimum at $\mathbf{k}=0$.

The magnon spectrum of the perpendicular magnetized ultra-thin ferromagnetic film can be written as [29]:

$$
\omega_{k}=\left\{\left[\omega_{H}+\omega_{e x}(a k)^{2}\right]\left[\omega_{H}+\omega_{e x}(a k)^{2}+\omega_{M} f(k \tau)\right]\right\}^{1 / 2},
$$

where $\gamma=2 \pi 2.8 \mathrm{MHz} /$ Oe is the gyromagnetic ratio, $\omega_{H}=\gamma H_{i}, H_{i}=H_{e}-4 \pi M_{s}+H_{\perp}$ is an effective internal magnetic field, $H_{\perp}$ is the perpendicular anisotropy field, $M_{s}=139$ Oe is the saturation magnetization, $\omega_{M}=4 \pi \gamma M_{s}=2 \pi \times 4.9 \mathrm{GHz}, \omega_{e x} a^{2}=2 \pi \times 1.09 \times 10^{-2}$ $\mathrm{Hz} \mathrm{cm}^{2}$ is the exchange constant, $f(k \tau)=1-[1-\exp (-k \tau)] / k \tau, \tau$ is the thickness of the film. All numerical parameters are given for YIG. Spin waves are assumed to be propagated only in the film plane and there is a uniform magnetization along the depth.

The critical density of pumped magnons at temperature $T$ can be estimated by the following equation:

$$
\frac{N_{p}}{A_{s}}=\frac{N(\mu, T)}{A_{s}}-\frac{N(0, T)}{A_{s}}
$$

where the sample volume is replaced by the film area $A_{s}$. The Eq.(23) in the high-population approximation has the form:

$$
\frac{N_{p}}{A_{s}} \approx \frac{k_{B} T}{2 \pi} \int_{0}^{k_{T}}\left(\frac{1}{\hbar \omega_{k}-\mu}-\frac{1}{\hbar \omega_{k}}\right) k d k,
$$

where $k_{T}$ corresponds to the frequency $\omega_{k} \simeq k_{B} T / \hbar$. This integral at $\mu \rightarrow \hbar \omega_{0}$ has a logarithmic divergence for an infinitely large film. However, for a finite film we have a magnetostatic mode on the bottom, which can be separated from the spin-wave spectrum by a small gap $\Delta \omega$. Thus, we can estimate Eq.(24) as

$$
\frac{N_{p}}{A_{s}} \approx \frac{k_{B} T}{4 \pi \hbar \omega_{e x} a^{2}} \ln \left(\frac{\omega_{0}}{\Delta \omega}\right)
$$

Note that this formula corresponds to the model of ultra-thin film, in which the magnon dynamics is considered in two dimensions. Magnetic excitations across the film plane are 
discrete and they interact weakly with magnons in the plane of the film. For this reason they practically do not affect the quasi-equilibrium in the two-dimensional system. Formula (25) is convenient for simple estimates, which can be refined by numerical calculations with accounting for all magnetic degrees of freedom.

\section{Critical angle}

Let us now find a critical angle of the magnetic moment deviation from the equilibrium, which is assumed to correspond to the critical number of excited magnons. This angle is defined by the ratio of perpendicular spin component to its longitudinal component $\tan \theta=$ $S_{\perp} / S_{z}$. The perpendicular component is equal to

$$
\begin{aligned}
S_{\perp} & =\sqrt{S_{x}^{2}+S_{y}^{2}}=\sqrt{\frac{S_{+} S_{-}+S_{-} S_{+}}{2}} \\
& \approx \sqrt{2 S a^{*} a}=\sqrt{2 S N_{p}} .
\end{aligned}
$$

Substituting $S_{z} \simeq S$, for small angles one obtains

$$
\theta \approx \sqrt{\frac{2 N_{p}}{S}}=\sqrt{\frac{2 \hbar \gamma}{M_{s}} \frac{N_{p}}{A_{s} \tau}} .
$$

For the film thickness $\tau=1 \mu \mathrm{m}, \omega_{0}=2 \pi \times 2.5 \mathrm{GHz}, \Delta \omega=2 \pi \times 1 \mathrm{~Hz}$ at room temperature $T=300 \mathrm{~K}$ we have $\theta_{\text {film }} \approx 0.044\left(2.5^{\circ}\right)$. An estimate for a bulk material at the same conditions gives $\theta_{\text {bulk }} \approx 0.061\left(3.5^{\circ}\right)$. Taking into account finite thickness of the film, one can expect the experimental value of the angle will be within $\theta_{\text {film }}<\theta<\theta_{b u l k}$. For comparison, the BEC deflection angle in the antiferromagnetic liquid crystal ${ }^{3} \mathrm{He}-\mathrm{B}$ was about $10^{-3}$ [1].

\section{BEC STABILITY}

The critical density is required but not a sufficient condition for a uniform magnon BEC. For the BEC stability it is important to consider the interaction between magnons. The Hamiltonian of magnon system described by creation $\left(b_{\mathbf{k}}^{\dagger}\right)$ and annihilation $\left(b_{\mathbf{k}}\right)$ bose operators can be written in the form:

$$
\mathcal{H}=\sum_{\mathbf{k}}\left(\varepsilon_{\mathbf{k}}-\mu\right) b_{\mathbf{k}}^{\dagger} b_{\mathbf{k}}+\mathcal{H}_{4}+\mathcal{H}_{6}+\mathcal{H}_{8}+\ldots
$$


where the magnon-magnon interaction terms are

$$
\begin{aligned}
& \mathcal{H}_{4}=\frac{1}{2} \sum_{\mathbf{1 , 2 ; 3}, \mathbf{3}} \Phi_{4}\left(\mathbf{k}_{1}, \mathbf{k}_{2} ; \mathbf{k}_{3}, \mathbf{k}_{4}\right) b_{\mathbf{1}}^{\dagger} b_{\mathbf{2}}^{\dagger} b_{\mathbf{3}} b_{4} \Delta\left(\mathbf{k}_{1}+\mathbf{k}_{2}-\mathbf{k}_{3}-\mathbf{k}_{4}\right), \\
& \mathcal{H}_{6}=\frac{1}{3} \sum_{\mathbf{1}, \mathbf{2}, \mathbf{3} ; \mathbf{4}, \mathbf{5}, \mathbf{6}} \Phi_{6}\left(\mathbf{k}_{1}, \mathbf{k}_{2}, \mathbf{k}_{3} ; \mathbf{k}_{4}, \mathbf{k}_{5}, \mathbf{k}_{6}\right) b_{\mathbf{1}}^{\dagger} b_{\mathbf{2}}^{\dagger} b_{\mathbf{3}}^{\dagger} b_{\mathbf{4}} b_{\mathbf{5}} b_{\mathbf{6}} \\
& \times \Delta\left(\mathbf{k}_{1}+\mathbf{k}_{2}+\mathbf{k}_{3}-\mathbf{k}_{4}-\mathbf{k}_{5}-\mathbf{k}_{6}\right)
\end{aligned}
$$

and so fourth. According to Bogoliubov's theory (see, e.g., [2], [30]), we have to single out classical condensate amplitudes with $\mathbf{k}=\mathbf{0}: b_{\mathbf{0}}^{\dagger}=b_{\mathbf{0}}=\sqrt{N_{0}}, N_{0}=N-N^{\prime}$. Here $N=$ $\sum_{\mathbf{k}} b_{\mathbf{k}}^{\dagger} b_{\mathbf{k}}$ is the total magnon number and $N_{0}$ is the magnon number in the condensate. Assuming that $N^{\prime} / N \ll 1$, we can reduce the Hamiltonian (28) to $\mathcal{H}=\mathcal{H}_{0}+\mathcal{H}_{2}$, where

$$
\mathcal{H}_{0}=\left(\varepsilon_{\mathbf{0}}-\mu\right) N_{\mathbf{0}}+\frac{1}{2} \mathcal{T}_{4}(0) N_{0}^{2}+\frac{1}{3} \mathcal{T}_{60} N_{0}^{3}+\ldots
$$

is the condensate energy and

$$
\mathcal{H}_{2}=\sum_{\mathbf{k} \neq \mathbf{0}}\left[A_{\mathbf{k}} b_{\mathbf{k}}^{\dagger} b_{\mathbf{k}}+\frac{B_{\mathbf{k}}}{2}\left(b_{\mathbf{k}} b_{-\mathbf{k}}+b_{\mathbf{k}}^{\dagger} b_{-\mathbf{k}}^{\dagger}\right)\right] .
$$

Here

$$
\begin{aligned}
A_{\mathbf{k}} & =\varepsilon_{\mathbf{k}}-\mu+2 \mathcal{T}_{4}(\mathbf{k}) N_{0}+3 \mathcal{T}_{6}(\mathbf{k}) N_{0}^{2}+\ldots \\
\mathcal{T}_{4}(\mathbf{k}) & =\Phi_{4}(\mathbf{k}, \mathbf{0} ; \mathbf{k}, \mathbf{0}) \\
\mathcal{T}_{6}(\mathbf{k}) & =\Phi_{6}(\mathbf{k}, \mathbf{0}, \mathbf{0} ; \mathbf{k}, \mathbf{0}, \mathbf{0}) \ldots
\end{aligned}
$$

and

$$
\begin{aligned}
B_{\mathbf{k}} & =\mathcal{S}_{4}(\mathbf{k}) N_{0}+\mathcal{S}_{6}(\mathbf{k}) N_{0}^{2}+\ldots \\
\mathcal{S}_{4}(\mathbf{k}) & =\Phi_{4}(\mathbf{k},-\mathbf{k} ; \mathbf{0}, \mathbf{0}) \\
\mathcal{S}_{6}(\mathbf{k}) & =\Phi_{6}(\mathbf{k},-\mathbf{k}, \mathbf{0} ; \mathbf{0}, \mathbf{0}, \mathbf{0}) \ldots
\end{aligned}
$$

Diagonalizing the quadratic form (30) by the linear canonical transformation $b_{\mathbf{k}}=u_{\mathbf{k}} c_{\mathbf{k}}+$ $v_{\mathbf{k}} c_{-\mathbf{k}}^{\dagger}$, we find that

$$
\mathcal{H}_{2}=U_{2}+\sum_{\mathbf{k} \neq \mathbf{0}} \widetilde{\varepsilon}_{\mathbf{k}} d_{\mathbf{k}}^{\dagger} d_{\mathbf{k}}
$$

where

$$
U_{2}=\frac{1}{2} \sum_{\mathbf{k} \neq \mathbf{0}}\left(\widetilde{\varepsilon}_{\mathbf{k}}-A_{\mathbf{k}}\right)
$$


and

$$
\widetilde{\varepsilon}_{\mathbf{k}}=\operatorname{sign}\left(B_{\mathbf{k}}\right)\left(A_{\mathbf{k}}^{2}-B_{\mathbf{k}}^{2}\right)^{1 / 2}
$$

is the spectrum of quasiparticles. From this formula obviously follows the following criterion of the condensate stability:

$$
B_{\mathbf{k}}>0, \quad A_{\mathbf{k}}^{2}-B_{\mathbf{k}}^{2}>0
$$

For the theory with four-magnon interactions we obtain

$$
\mathcal{S}_{4}(\mathbf{k})>0, \quad\left[2 \mathcal{T}_{4}(\mathbf{k})\right]^{2}-\left[\mathcal{S}_{4}(\mathbf{k})\right]^{2}>0
$$

If this condition does not work (for an attractive interaction, $\mathcal{S}_{4}(\mathbf{k})<0$ ), the six-magnon interactions which are repulsive in ferro- and antiferromagnets (due to specificity of HolsteinPrimakoff representation of spin operators by the bose operators) can make the system stable:

$$
\begin{aligned}
\mathcal{S}_{4}(\mathbf{k})+\mathcal{S}_{6}(\mathbf{k}) N & >0 \\
{\left[2 \mathcal{T}_{4}(\mathbf{k})+3 \mathcal{T}_{6}(\mathbf{k}) N\right]^{2}-\left[\mathcal{S}_{4}(\mathbf{k})+\mathcal{S}_{6}(\mathbf{k}) N\right]^{2} } & >0
\end{aligned}
$$

In this case one can expect a sharp appearence of the uniform condensate at $N>$ $-\mathcal{S}_{4}(\mathbf{k}) / \mathcal{S}_{6}(\mathbf{k})$. A detailed analysis will be published elsewhere.

\section{DISCUSSION}

The Bose-Einstein condensate of magnons with $k=0$ is a uniform precession of the magnetic moment in the effective magnetic field. How does this precession differ from the usual precession of the magnetic moment? It is known that the uniform precession of a magnetic moment deviated from equilibrium precesses in an effective field and is gradually damped due to losses in the magnetic material and radiation damping. In this case we have an excited coherent state of magnons with $k=0$, and the magnons of the entire spectrum are in thermodynamic equilibrium with the chemical potential $\mu=0$. In the case of BEC, we also have an excited coherent state of magnons with $k=0$, but this state arose as a result of a change in the density of magnons and their chemical potential becomes $\mu=\varepsilon_{0}$. In this case, the losses of the precessing condensate in the magnetic material disappears, the spin superfluidity arises. There remains only a weak radiation damping [19, 21], which leads 
to a long-lived coherent precession. This long-lived state was first observed experimentally in antiferromagnetic ${ }^{3} \mathrm{He}-\mathrm{B}[3]$.

In this paper we have focused on critical conditions of BEC in ferro- and antiferromagnets, bulk and thin films. Recent similar analysis [31] demonstrated a good agreement with experiments for nuclear magnon BEC. Let us list main results.

1. We have shown that in general the only highly populated region of the spectrum (which has been previously considered for particular cases in Refs. [1], [28]) is responsible for the formation of any BEC. The high population approximation, $n_{\mathbf{k}} \simeq k_{B} T /\left(\varepsilon_{\mathbf{k}}-\mu\right)$, however, does not mean that we deal with the classical physics, this is an expression for the Bose-Einstein distribution if $n_{\mathbf{k}} \gtrsim 1$. The opposition of high density of particles or quasiparticles to their high population makes the classical criterion of Maxwell-Boltzmann statistics inapplicable which means that the Bose-Einstein condensation condition $\mu=\min \varepsilon_{\mathbf{k}}$ always occurs in the degenerate bose gas. The high population approximation substantially simplifies analysis of the critical conditions and can simplify magnetic dynamics simulations of systems with BEC by the use of classical variables instead of operators. So far as the BEC occurs in the $k$-space, the most convenient form of simulation is the use of kinetic equations for $n_{\mathbf{k}}$ with the integrals of magnon-magnon collisions (see, e.g., (2) ), sources of pumping and relaxation [19], [12].

2. We have found that the condition of magnon BEC in one of the most interesting materials, perpendicular magnetized YIG thin film, is fulfilled at a small angle, when signals are usually treated as excited spin waves [32].

3. We have estimated that in hematite, high-temperature antiferromagnet, the BEC should occur at much lower level of the magnon excitation compared to that of ferromagnetic YIG. We believe that this theoretical prediction can open a new direction of purposeful studies for fundamental and applied research.

4. We have generalized the Bogoliubov's BEC theory to the case of multi-particle interactions. According to this theory the uniform Bose-Einstein condensate is unstable if the four-particle interaction is attractive. This situation sometimes takes place in ferro- and antiferromagnets. The account of six-magnon interactions (which are repulsive in magnets) can resolve the problem of BEC stability. In principle, there are also factors of time, the sample size, and relaxation for the BEC instability has to be developed in the system.

One more fundamental problem that remains in this field is to connect microscopic and 
phenomenological description of bose system with Bose-Einstein condensate. This problem exists for a long time and it's solution, say, on the base of magnon systems, will advance the understanding and progress in all areas where the BEC is manifested. The solution of this problem will help understand the spin superfluidity as a property of the magnetic system that accompanies BEC of quasi-equilibrium magnons.

In conclusion, we emphasize that the Bose-Einstein condensation of quasi-equilibrium magnons is a fundamental law of physics. BEC appears due to quantum statistics of quasiparticles in magneto-ordered systems and can exist at room, or even higher temperatures. One of the most intriguing properties of the BEC is a superfluid spin current, a coherent quantum flow of energy and information. This understanding of magnon BEC in different magnetic materials can be very useful for spin transport and magnonic quantum devices. An interest in the spin currents, the magnetization projection transfer in magnetic materials, is growing every year.

The authors wish to thank Grigory Volovik and anonimous reviewer for helpful comments. For Yu. M. B. this work was financially supported by the Russian Science Foundation (grant RSF 16-12-10359).

[1] Yu. M. Bunkov, G. E. Volovik, Spin superfluidity and magnon BEC, Chapter IV of the book "Novel Superfluids", eds. K. H. Bennemann and J. B. Ketterson (Oxford, University press, 2013)

[2] V. L. Safonov, Nonequilibrium Magnons (Wiley-VCH, Weinheim, 2012).

[3] A. S. Borovik-Romanov, Yu. M. Bunkov, V. V. Dmitriev, Yu. M. Mukharskiy, JETP Lett. 40 (1984) 1033.

[4] I. A. Fomin, JETP Lett. 40 (1984) 1037.

[5] L. Pitaevskii and S. Stringari, Bose-Einstein condensation (Clarendon Press, Oxford, 2003)

[6] G. E. Volovik, J. Low Temp. Phys. 153 (2008) 266.

[7] Yu. M. Bunkov and G. E. Volovik, J. Low Temp. Phys. 150 (2008) 135; J. Phys.: Condens. Matter 22 (2010) 164210.

[8] Yu. M. Bunkov, J. Phys.: Condens. Matter 21 (2009) 164201.

[9] T. Sato , T. Kunimatsu , K. Izumina , A. Matsubara, M. Kubota, T. Mizusaki and Yu. M. 
Bunkov, Phys. Rev. Lett. 101 (2008) 055301.

[10] P. Hunger, Yu. M. Bunkov, E. Collin and H. Godfrin, J. Low Temp. Phys. 158 (2010) 129.

[11] S. O. Demokritov, V. E. Demidov, O. Dzyapko, G. A. Melkov, A. A. Serga, B. Hillebrands, and A. N. Slavin, Nature 443 (2006) 430.

[12] D. A. Bozhko, A. A. Serga, P. Clausen, V. I. Vasyuchka, F. Heussner, G. A. Melkov, A. Pomyalov, V. S. Lvov and B. Hillebrands, Nature Physics 12 (2016) 1057.

[13] Yu. M. Bunkov, P. M. Vetoshko, I. G. Motygullin, T. R. Safin, M. S. Tagirov and N. A. Tukmakova, Mag. Res. in Solids 17 (2015) 15205.

[14] Yu. M. Bunkov, Physics Uspekhi 53 (2010) 848.

[15] Yu. M. Bunkov, E. M. Alakshin, R. R. Gazizulin, A. V. Klochkov, V. V. Kuzmin, V. S. L'vov, M. S. Tagirov, Phys. Rev. Lett. 108 (2012) 177002.

[16] M. Borich, Yu. M.Bunkov, M. I.Kurkin, A. P. Tankeev, JETP Lett. 105 (2017) 24.

[17] Yu. M. Bunkov, J. Low Temp. Phys. 185 (2016) 399.

[18] Yu. D. Kalafati and V. L. Safonov, Sov. Phys. JETP 68 (1989) 1162.

[19] Yu. D. Kalafati and V. L. Safonov, JETP Lett. 50 (1989) 149; Sov. Phys. JETP 73 (1991) 836.

[20] V. L. Safonov. Physica A 188 (1992) 675.

[21] Yu. D. Kalafati and V. L. Safonov, J. Magn. Magn. Mater. 123 (1993) 184.

[22] A. V. Lavrinenko, V. S. L'vov, G. A. Melkov, and V. B. Cherepanov, Sov. Phys. JETP 54 (1981) 542 .

[23] G. A. Melkov, V. L. Safonov, A. Yu. Taranenko, and S. V. Sholom, J. Magn. Magn. Mater. 132 (1994) 180.

[24] S. A. Govorkov and V. A. Tulin, Sov. Phys. JETP 68 (1989) 807.

[25] V. E. Demidov, O. Dzyapko, S. O. Demokritov, G. A. Melkov, and A. N. Slavin, Phys. Rev. Lett. 99 (2007) 037205.

[26] A. V. Chumak, G. A. Melkov, V. E. Demidov, O. Dzyapko, V. L. Safonov, and S. O. Demokritov, Phys. Rev. Lett. 102 (2009) 187205.

[27] K. Huang, Introduction to statistical physics (Taylor and Francis, New York, 2001).

[28] A. Rückriegel and P. Kopietz, Phys. Rev. Lett. 115 (2015) 157203.

[29] G. N. Kakazei, P. E. Wigen, K. Yu. Guslienko, V. Novosad, A. N. Slavin, V. O. Golub, N. A. Lesnik, and Y. Otani, Appl. Phys. Lett. 83 (2004) 443. 
[30] N. N. Bogolubov and N. N. Bogolubov Jr., Introduction to quantum statistical mechanics (World Scientific Publishing Company, Singapore, 2009)).

[31] R. R. Gazizulin, Yu. M. Bunkov, V. L. Safonov, JETP Letters 102 (2015) 766.

[32] Yu. K. Fetisov, C. E. Patton, and V. T. Synogach, IEEE Trans. Magn. 35 (1999) 4511. 